\title{
SVD-based Separation of Stable and Inertial Cavitation Signals Applied to Passive Cavitation Mapping During HIFU
}

\author{
Parag V. Chitnis ${ }^{1}$, Member, IEEE, Caleb H. Farny ${ }^{2}$, and Ronald A. Roy ${ }^{3}$ \\ ${ }^{1}$ Department of Bioengineering, George Mason University, Fairfax, VA 22030 \\ ${ }^{2}$ Department of Mechanical Engineering, Boston University, Boston, MA 02215 \\ ${ }^{3}$ Department of Engineering Science, University of Oxford, Oxford OX1 3PJ, UK
}

\begin{abstract}
Detection of inertial and stable cavitation is important for guiding high-intensity focused ultrasound (HIFU). Acoustic transducers can passively detect broadband noise from inertial cavitation and the scattering of HIFU harmonics from stable cavitation bubbles. Conventional approaches to cavitation noise diagnostics typically involve computing the Fourier transform of the time domain noise signal, applying a custom comb-filter to isolate the frequency components of interest, followed by an Inverse-Fourier transform. We present an alternative technique based on singular value decomposition (SVD) that efficiently separates the broadband emissions and HIFU harmonics. Spatio-temporally resolved cavitation detection was achieved using a 128element, 5-MHz linear-array ultrasound imaging system operating in receive mode at 15 frames/s. A 1.1-MHz transducer delivered HIFU to tissue-mimicking phantoms and excised liver tissue for a duration of $5 \mathrm{~s}$. Beamformed radiofrequency signals corresponding to each scan line in a frame were assembled into a matrix and SVD was performed. Spectra of the singular-vectors obtained from a tissue-mimicking gel phantom were analyzed by computing the peak ratio $(R)$, defined as the ratio of the peak of its $5^{\text {th }}$-order polynomial fit and the maximum spectral peak. Singular-vectors that produced a $R<0.048$ were classified as those representing stable cavitation, i.e., predominantly containing harmonics of HIFU. The projection of data onto this singular-base reproduced stable-cavitation signals. Similarly, singular-vectors that produced a $R>0.2$ were classified as those predominantly containing broadband noise associated with inertial cavitation. These singular-vectors were used to isolate the inertial-cavitation signal. The R-value thresholds determined using gel data were then employed to analyze cavitation data obtained from bovine liver ex vivo. The SVD-based method faithfully reproduced the structural details in the spatiotemporal cavitation maps produced using the more cumbersome comb-filter approach with a maximum root-mean-squared error of $10 \%$.
\end{abstract}

Key words: HIFU, cavitation, SVD, filtering

\section{Introduction}

Acoustic cavitation, broadly defined as the nucleation and/or forced oscillations of a gas bubble in the presence of a sound field, plays a significant role in many ultrasoundmediated therapeutic processes. Inertial cavitation, which refers to the unstable growth and rapid violent collapse of a microbubble, can enhance tissue heating from high intensity focused ultrasound (HIFU) and may play an important role in ultrasound-mediated thermal ablation of tumors [1-4]. Stable cavitation is the 
repetitive volumetric pulsation of gas-filled microbubbles about an equilibrium radius. The pulsations are typically nonlinear and often induce microstreaming flows that promote mass transport on micron scales [5, 6]. Mechanical effects from cavitation activity have been shown to play vital roles in facilitating the opening of the blood-brain barrier [7-9], inducing sonothrombolysis [10-13], tissue fractionation via histotripsy $[11,14-16]$, enhancing drug delivery [17$19]$, in addition to tumor ablation $[8,20]$. In all of these applications, real time determination of the location, timing, magnitude, and type of the cavitation activity is of great importance.

Cavitation dynamics can be probed using two types of techniques, active detection and passive detection. Active detection typically employs focused, narrow-band high-frequency transducers confocally positioned with the HIFU field. The active detector emits a pulse-train timed to arrive in the HIFU focal volume coincident with the lower-frequency HIFU sonication pulse. The presence and oscillations of microbubbles result in a corresponding amplitude modulation of the backscattered signal $[21,22]$. This method provides high sensitivity and precise localization of cavitation events.

Alternately, cavitation activity can be detected directly by sensing the acoustic emissions produced by oscillating microbubbles using passive acoustic sensors. Acoustic signals from stable cavitation bubbles typically consist of harmonics and sub/ultra-harmonics of the incident sound field; these emissions can result from scattering of the (typically nonlinear) HIFU field [1, 23, 24] and from nonlinear bubble oscillations [25, 26]. In comparison, inertial cavitation collapse is transient and results in broadband acoustic emissions [24, 27]. The process of delineating cavitation type based on the noise spectrum is typically called cavitation noise diagnostics, and the process of sampling the noise field using one or more acoustic sensors is termed passive cavitation detection (PCD).

The utility of cavitation detection applied to therapeutic ultrasound has progressed as the capability of applicable hardware and software tools have evolved. This has eased logistical issues germane to clinical use, such as constraints on the orientation of the cavitation detector, improvement in the detector spatial resolution and sensing volume, and enhanced ability to delineate cavitation type via noise diagnostics. The simplest and most common PCD employs a single-element transducer operated in passive mode $[1,3,7$, $8,10,11,13,14,16-18,20,23,28-30]$, generally with a fixed geometric focus. When tightly focused, these detectors offer high sensitivity in detecting cavitationinduced emissions originating from its focal region. They are usually employed noninvasively and the sensing volume is usually much smaller than the HIFU therapy volume. Processing the signal from a single-element PCD is simple and fast. However, it is basically a point detector, and not suitable for spatially mapping cavitation activity.

More recently, the architecture of clinical-grade ultrasound imaging systems has become more accessible such that these normally active systems can be operated in passive mode, facilitating their use as arraybased active and passive cavitation detection systems. Some active schemes employ Bmode images [23] and speckle-tracking correlation techniques [31] to detect the hyperechoic nature of high-temperature boiling cavitation regions, but are ill equipped to sense inertial cavitation events or discrete stable cavities due to limitations in SNR and spatial resolution. As hardware and software capabilities have advanced, the 
acquisition of high-resolution radiofrequency $(\mathrm{RF})$ data from individual array elements has improved, leading to sophisticated signal processing schemes customized for cavitation detection and noise diagnostics.

One straightforward approach for sensing inertial cavitation activity is to apply a digital high-pass filter to the RF signals from the array $[28,32]$. A number of groups [24, 29, 30, 33-35] have employed digital comb filters to isolate the broadband emissions from the HIFU harmonics; carefully-tuned band-reject comb filters yield broadband energy (inertial cavitation) whereas bandpass comb filters reject the bulk of the broadband noise and isolate the energy in the harmonics (stable cavitation). The delineation of cavitation type comes from the frequency-domain pre-filter, and the spatial map of a given type of cavitation activity comes from the delay-sum-integrate beamformer.

All the techniques described above rely on the application of a frequency domain filter to isolate features of the noise field that are associated with different types of cavitation. This requires careful consideration of the HIFU center frequency, bandwidth, and nonlinearity as well as the spectral response of the PCD. The HIFU field properties are specific to the transducer employed, and can also change depending on peak HIFU pressure amplitude, tissue properties and temperature. This reliance on detailed a priori knowledge of the expected noise field complicates implementation and makes it difficult to design PCD systems that can be applied to multiple scenarios. As with the single element detector, one major drawback to such a processing scheme is that it must be carefully "tuned" to the application at hand [36]. Whether the processing is performed in the frequency domain or via time-based finite impulse response filters, choosing the rejection bands has a degree of subjectivity due to the inherent overlap of the broadband emissions with the HIFU harmonics and underlying system electronic noise. As a result, a certain level of inaccuracy will exist when the analysis seeks to attribute the received energy to different phenomena associated with the various frequency bands. This issue becomes even more challenging when multiple transmit frequencies are incorporated into a therapeutic system [8]. While significantly more computationallyintense and still dependent on an empirical parameter, a time-based sum-of-harmonics approach [36] has recently shown improved sensitivity and accuracy in detecting the spatial location of cavitation types when compared to a similar comb filter method.

An alternate approach to the frequencydomain processing methods described above is to use singular value decomposition (SVD). Recently, SVD of RF data was employed in conjunction with a high-framerate linear-array transducer to discriminate bubble clouds from tissue based on their different spatio-temporal motion and echogenicity for real-time pulse-echo monitoring of histotripsy [37]. This approach relied on the fact that SVD of multi-frame RF data (assembled in a 2D matrix, $M$ ) achieves decomposition in a spatio-temporal base as shown in Eq. 1:

$$
M=U \cdot S \cdot V^{*}
$$

where $U$ and $V$ are unitary matrices that contain the temporal and spatial singular vectors, respectively, arranged in the decreasing order of energy, and $S$ is the diagonal matrix containing the weights of the projections [38]. It was shown that tissue signals had a high level of coherence resulting in higher-order singular vectors whereas broadband signals originating from cavitation, which are spatio-temporally incoherent, yielded lower-order singular 
vectors. Filtering was achieved by projecting the RF data on a sub-base of singular vectors containing the signal of interest. In this manner, a SVD-based approach was shown to successfully discriminate between inertial-cavitation noise and other ultrasonic signals encountered during diagnostic imaging [37].

The SVD method also was shown to successfully detect stable cavitation events in a phantom flow arrangement populated with ultrasound contrast agents (UCA) [26, 39]. In these studies, the SVD allowed the energy in the subharmonic band for all cavitation events to be quantified. These studies also demonstrated that the SVDbased technique offered higher sensitivity in characterizing subharmonics associated with nonlinear bubble oscillations than the basic frequency-domain filtering approaches.

The goal of this study was to evaluate whether SVD analysis is an effective method for distinguishing between inertial and stable cavitation signatures obtained using passive-sensing method. The rationale for using SVD is that under a given set of experimental conditions (i.e., peak acoustic pressure, pulse duration, etc.) cavitation events can be interpreted as identifiable realizations of a seemingly random process. Therefore, SVD is a potentially viable tool for analyzing and classifying microbubble activity in a manner that can be readily automated for analyzing large data sets of cavitation activity [38].

The implementation in this work is distinct from Arnal et al. [37], in that they used SVD-based scheme to differentiate between ultrasound-backscatter (active monitoring) signal from microbubbles and backscatter from tissue based on differences in spatio-temporal dynamics. In comparison, the present effort applied SVD-based processing to differentiate between harmonic and broadband signatures observed during HIFU-induced cavitation using a passive sensor-array.

Well-characterized beamformed RF data obtained using a linear-array PCD in a previous study [24, 40] was used to validate the SVD-based approach. In the previous study, the digitized RF data was analyzed using a comb filter designed to separate the harmonics generated by the HIFU source from the broadband energy present in the PCD signal. This paper will focus on development and implementation of an SVD-based processing scheme on the same data and its comparison with the more conventional comb filter approach.

\section{Methods}

Processing scheme and analysis described in this section were applied to cavitation data acquired in a previous study [24]. PCD experiments were performed using a tissuemimicking gel phantom, a bovine liver sample that was degassed for 30 minutes, and a bovine liver sample that was degassed for 60 minutes. Details of PCD data acquisition and signal-processing using the comb-filter approach are presented in [24]. A concise description is provided here for completeness. All signal-processing steps described below were performed using MATLAB (Mathworks, Natick, USA).

\section{A. Passive cavitation mapping}

FUS was delivered using a 1.1-MHz singleelement transducer (H102, Sonic Concepts) with a focal length of $62 \mathrm{~mm}$ and an aperture of $70 \mathrm{~mm}$. The focal peak acoustic pressures for experiments with the phantom, liver degassed for $30 \mathrm{~min}$, and the liver degassed for $60 \mathrm{~min}$ were $3.5 \mathrm{MPa}, 6.5$ $\mathrm{MPa}$, and $7.4 \mathrm{MPa}$, respectively. The resulting cavitation activity was monitored for approximately $5 \mathrm{~s}$ using a 128-element linear array (L10-5) that was operated with an ultrasound system (Terason 2000). The 
image plane of the linear array was oriented parallel to the acoustic axis of the FUS transducer. When working with an imaging array it is important to acknowledge the level of control over the beamforming process. Some systems allow acquisition of the RF signals prior to beamforming, and therefore the ability for the user to beamform the image. The system used in this study already applied a beamforming algorithm prior to acquisition of the RF data. RF data containing cavitation signatures were beamformed using a standard delayand-sum algorithm. Temporal sampling of PCD-based cavitation monitoring was determined by the frame rate of the Terason system, which was 15 FPS.

\section{B. Comb-filter-based signal processing}

The signals received by the PCD can be classified into three categories [41]: 1) fundamental frequency of the FUS source and its harmonics scattered by bubbles undergoing stable cavitation or vapor bubbles, 2) sub- and ultra-harmonics of the FUS frequency generated by stable cavitation, and 3) broadband acoustic emissions generated by inertial cavitation.

Isolation of broadband signal from harmonics required a carefully designed comb filter that was applied in the frequency domain. The time domain signal was Fourier transformed and a filter was applied by deleting a $150 \mathrm{kHz}$ band around each harmonic peak $(n f$, for $\mathrm{n}=1,2, \ldots, 12$ and $f$ is the fundamental frequency) and a $50 \mathrm{kHz}$ band around each ultra-harmonic peak (where the $p / 2, q / 3$ ultra-harmonics were considered, for $p=\mathrm{n}(\mathrm{n} \bmod 2 \neq 0)$ and $\mathrm{q}=$ $\mathrm{n}(\mathrm{n} \bmod 3 \neq 0))$ across the entire frequency range. The removed harmonic bands were replaced with signal sampled from windows adjacent to the harmonic bands thus preserving only the broadband spectral energy associated with inertial cavitation. Similarly, subtracting the filtered broadband spectrum from the original unfiltered spectrum yielded a spectrum consisting of harmonics associated with stable cavitation.

Inverse FFT was performed on inertial and stable cavitation spectra and the resulting time domain signals were further filtered using a six-stage high-pass Butterworth digital filter with a cut-off frequency of $1.5 \mathrm{MHz}$ to remove the fundamental FUS signal. Finally, the rootmean-squared voltage (equivalent to cumulative energy) of each inertial and stable cavitation RF line was computed, which provided separate spatio-temporal (vertical axis: lateral distance from FUStransducer focus; horizontal axis: sonication time) maps of inertial and stable cavitationnoise energy.

\section{SVD-based signal processing}

Beamformed RF data acquired for the data sets employed in SVD analysis contained 78 frames (5 seconds) of 2D (B-modeequivalent) data of $2572 \mathrm{RF}$ points (rows) in each of $128 \mathrm{RF}$ lines (columns). The data were combined into a 3D matrix of dimensions $2572 \times 128 \times 78$, and reshaped in to a $2 \mathrm{D}$ matrix with dimensions $2572 \times 9984$. SVD of the reshaped matrix resulted in a $2572 \times 2572$ matrix of singular-vectors. Spectra $\left(S_{n}(f), n=1: 2572\right)$ of singular-vectors were obtained by applying a Hanning window and performing an 8192-point Fast Fourier transform to each singular-vector. The spectrum of each singular-vector was normalized to the maximum value and fitted to a $5^{\text {th }}$-order polynomial using the polyfit function in MATLAB. The polynomial fit $\left(P_{n}(f)\right)$ closely tracked the magnitudefrequency profile of the broadband energy present in the singular-vectors. Spectra of the singular-vectors that contained significant energy corresponding to the harmonics of FUS exhibited sharp and distinct peaks at these harmonic frequencies. The polynomial fit did not track the sharp 
peaks associated with HIFU harmonics. Peak ratio, defined as $R_{n}=\max \left(P_{n}(f)\right) / \max \left(S_{n}(f)\right)$, was employed to identify singular-vectors that predominantly represented either harmonic signals associated with stable cavitation or broadband signals associated with inertial cavitation. A user-independent scheme to objectively classify a peak ratio and corresponding singular vector is described in Sec. E.

Time-domain signals that represented stable cavitation were separately recovered by projecting the raw RF data onto the base of singular vectors that corresponded to harmonic energy. Similarly, signals that represented inertial cavitation were separately recovered by projecting the raw RF data onto the base of singular vectors that represented broadband energy. Finally, the root-mean-squared voltage of each RF line was computed, which provided the separate spatio-temporal maps of inertial and stable cavitation activity similar to the comb-filter approach.

\section{Error analysis}

In addition to qualitative comparison between the spatio-temporal maps obtained by comb-filter and SVD approaches, quantitative error analysis was also performed. A $10-\mathrm{mm}$ (lateral) region (demarcated by the black dashed lines) within the spatio-temporal cavitation maps containing the majority of the cavitation signal was identified for each experimental result presented below. The spatial sum of the cavitation energy in this region provided cumulative cavitation energy as a function of sonication time. Root-mean-squared error (RMSE) was computed by comparing this energy-time profile obtained from the two methods for each experimental run.

\section{E. Pre-selection of peak-ratio threshold}

Minimization of error (described in Sec. D) between cavitation maps obtained from gelphantom data using frequency-comb filter and SVD analysis was used to determine the optimal threshold values of $R$ for identifying singular vectors corresponding to harmonic and broadband emissions. Based on this scheme, singular-vectors that produced $R<$ 0.048 were classified as those primarily representing harmonic signals associated with stable cavitation. Similarly, singularvectors that produced $R>0.2$ were classified as those representing broadband signals associated with inertial cavitation.

\section{Results}

\section{A. Comb-filter results}

Several examples of spatio-temporal maps of cavitation produced by the comb-filter approach are available in ref. [24]. An illustrative result obtained in a tissuemimicking gel phantom was reproduced by analyzing previously acquired cavitation data and is shown in Fig. 1; see ref. [24] for details regarding the measurement geometry and experimental methods employed. The region (lateral extent with respect to the HIFU focal distance) that contained the majority of cavitation activity is bounded by black dash lines. Unfiltered cavitation noise energy is shown in Fig. 1a. Comb filtering separated the map of stable cavitation activity (Fig. 1b) from inertial cavitation (Fig. 1c). The onset of inertial cavitation was observed when HIFU was turned on $(t \approx$ $0 \mathrm{~s})$, whereas significant harmonic noise associate with scattering from vapor cavities and stably oscillating microbubbles was not observed until $t>2 \mathrm{~s}$. Increase in stable cavitation activity also was accompanied by a reduction in observed inertial cavitation, which is likely due to formation of hot gasvapor bubbles that are less likely to undergo inertial collapse [40, 42]. In addition, persisting microbubbles can contribute to 
the acoustic-shielding phenomenon [23, 43, 44], which would further reduce inertial cavitation at the focus.

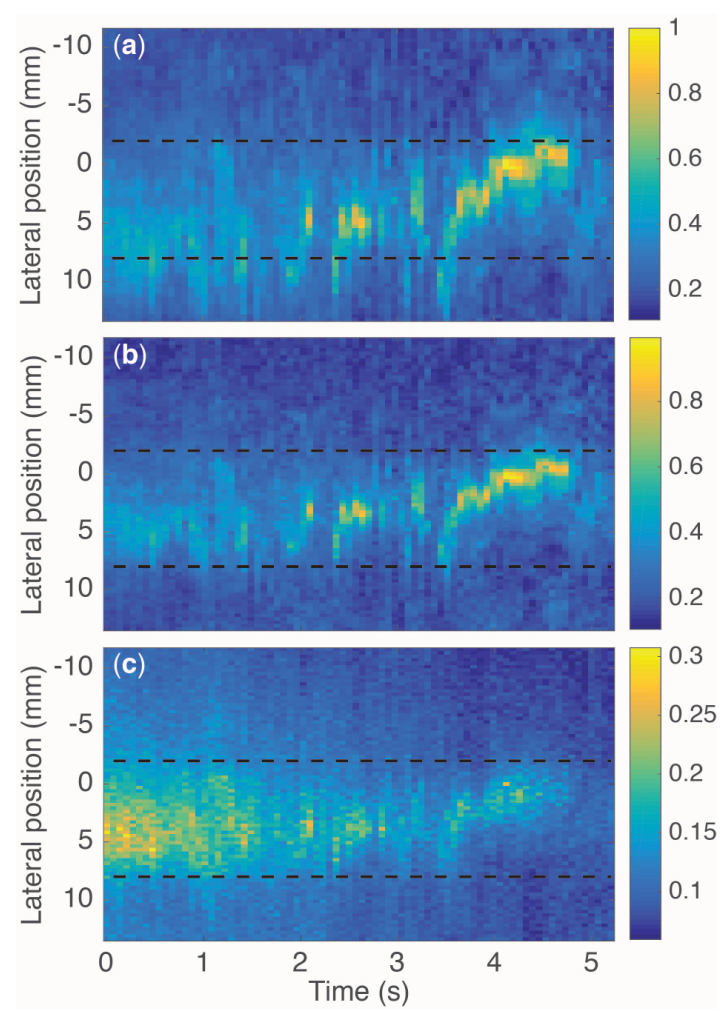

Figure 1. Spatio-temporal maps of cavitation-noise energy (RMS voltage normalized to the maximum value) measured in a tissue-mimicking gel phantom with the Terason system (a) unfiltered cavitation activity, (b) backscattered HIFU harmonics (indicative of stable cavitation), and (c) broadband noise associated with inertial cavitation. Harmonics and broadband maps were produced using the data and comb-filter approach presented in [1].

\section{B. SVD-based analysis}

The SVD-based processing was first performed on the same dataset [24] that was used to reproduce Fig. 1. The Fourier spectrum of a singular-vector (blue curve) that predominantly contains one HIFUharmonic signal and its $5^{\text {th }}$-order polynomial fit (red curve) are shown in Fig. 2a. Similarly, the spectrum of a singular-vector that contains significant broadband energy and its polynomial fit are shown in Fig. $2 b$. These figures illustrate that the peak ratio $R$, defined as the singular-vector spectral peak divided by the peak of the polynomial fit, is
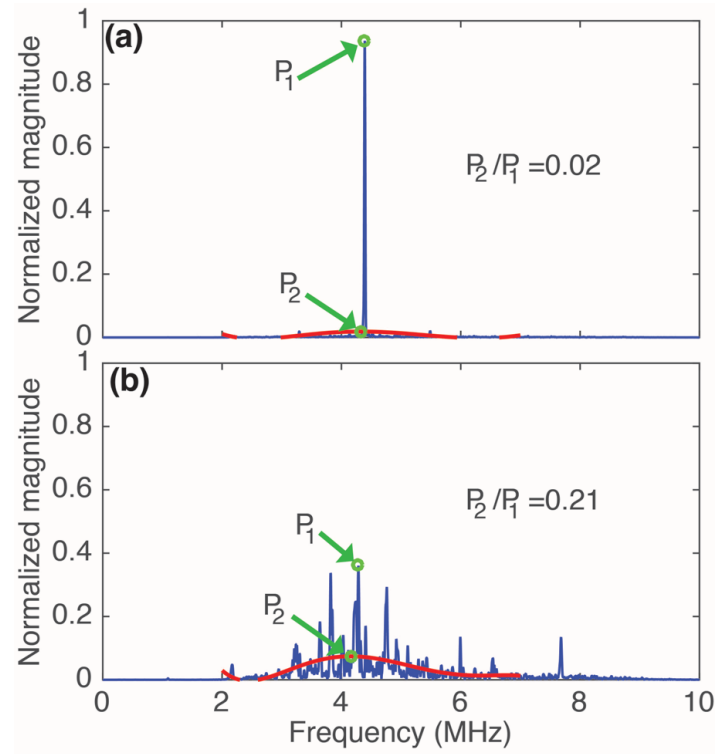

Figure 2. Scheme for classifying singular vectors as representing stable or inertial cavitation. (a) a representative spectrum of a singular vector that predominantly contains harmonic signal associated with stable cavitation. (b) a representative spectrum of a singular vector that predominantly contains broadband signal associated with inertial cavitation. Each spectrum was normalized with respect to the largest peak magnitude. The peak (P1) in each spectrum was compared to the peak of its 5-th order polynomial fit $\left(\mathrm{P}_{2}\right)$ to classify singular vectors as those predominantly containing harmonic or broadband signals.

an appropriate quantitative measure for differentiating between singular-vectors that represent harmonic energy and singular-

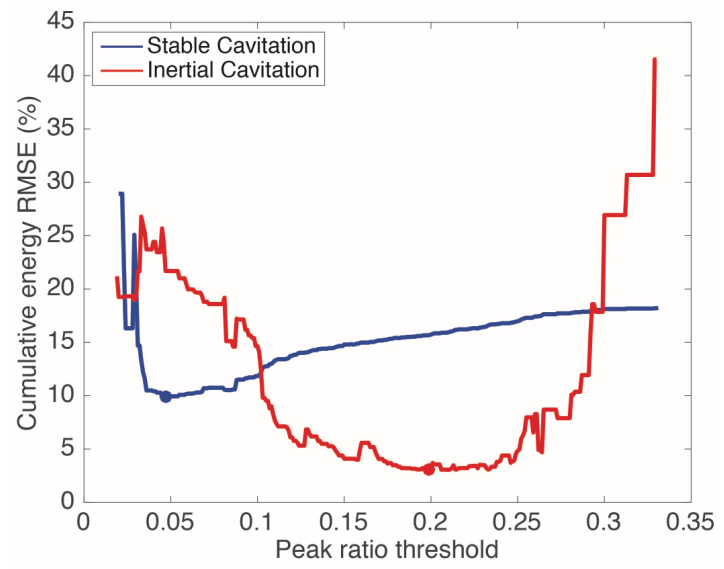

Figure 3. A plot of cavitation energy RMSE as a function of peak-ratio $(R)$ threshold used to classify singular vectors as those representing harmonic (stable) or broadband (inertial) cavitation signature. Minimization of error indicates that $\mathrm{R}<0.048$ and $\mathrm{R}>0.2$ optimally classify singular vectors for stable cavitation and inertial cavitation, respectively. 
vectors that represent broadband energy.

Figure 3 shows the RMSE obtained when comparing cavitation maps from phantom data for a range of $R$ threshold values. The lowest RMSE for stablecavitation maps was achieved when singular vectors with $R<0.048$ were classified as those primarily representing harmonic signals. Similarly, the lowest RMSE for broadband cavitation was achieved when singular-vectors that produced $R>0.2$ were
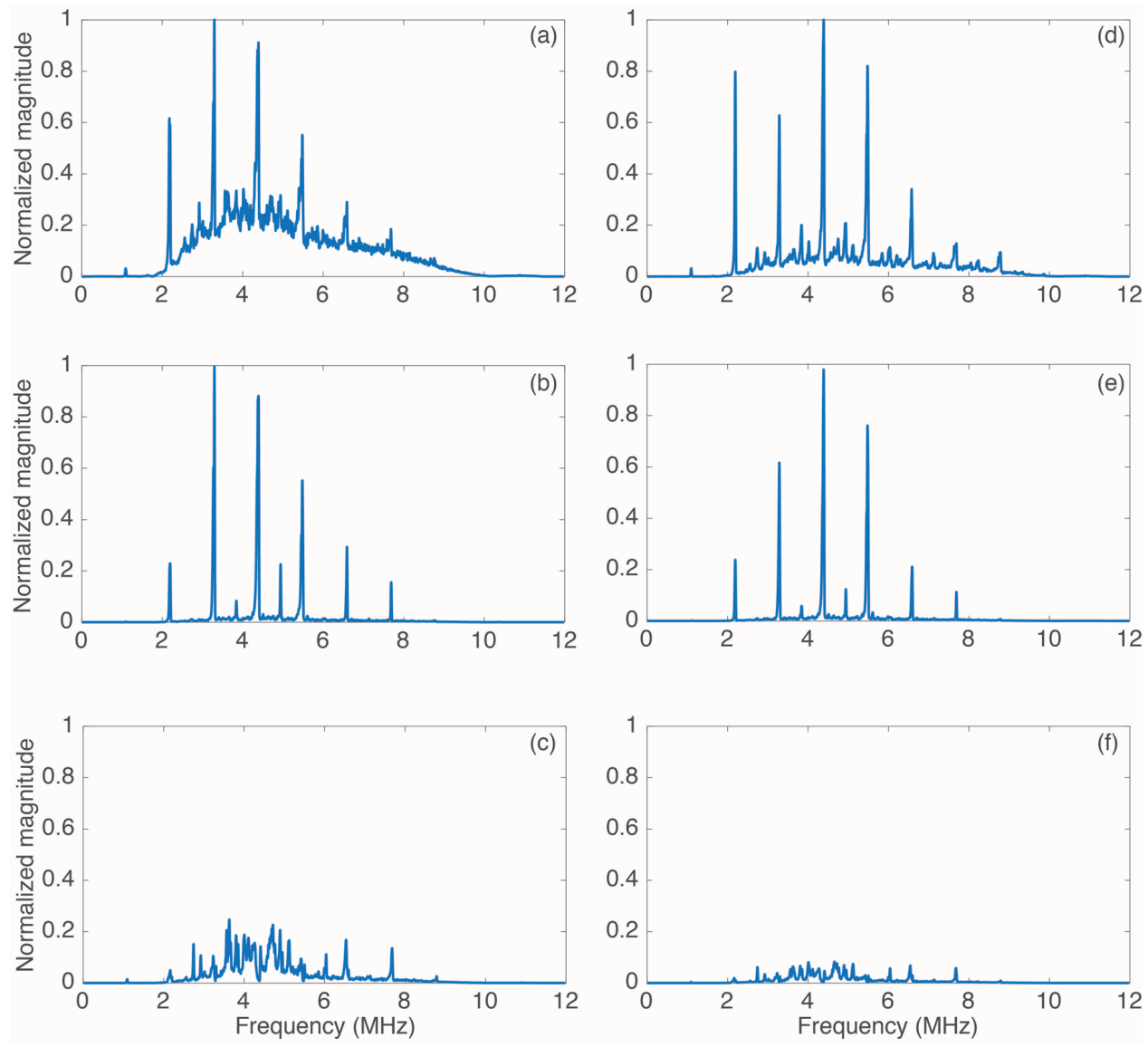

Figure 4. Average magnitude spectra of cavitation noise, calculated by averaging the spectra of radio-frequency signals obtained from the region bound by dash lines in Fig. 1 at two specific time points during the HIFU exposure. Spectra of unfiltered cavitation energy, SVD-derived harmonic energy, and SVD-derived broadband energy at Time $=0.5 \mathrm{~s}$ are shown in (a), (b), and (c), respectively. Spectra of unfiltered cavitation energy, SVD-derived harmonic energy, and SVDderived broadband energy at Time $=4.5 \mathrm{~s}$ are shown in (d), (e), and (f), respectively. Spectra at both time points were normalized by the peak magnitude of the spectrum obtained from the unfiltered data at that time point. SVD-based filtering successfully separated signals corresponding to stable and inertial cavitation activity.

classified as those representing broadband signals associated with inertial cavitation.

Radio-frequency (RF) data obtained from the region bound between dashed lines
SVD-based filtering removed broadband energy present in the original signal and the spectra of the filtered signals indicates the presence of predominantly HIFU harmonics. 
Figures $4 \mathrm{c}$ and $4 \mathrm{f}$ show spectra of the timedomain cavitation signals that were recovered by projecting the beamformed RF data onto the base of singular vectors that corresponded to broadband energy $(R>0.2)$. This spectrogram showed that the SVDbased filtering effectively removed the harmonic energy from the original cavitation signal leaving only the broadband
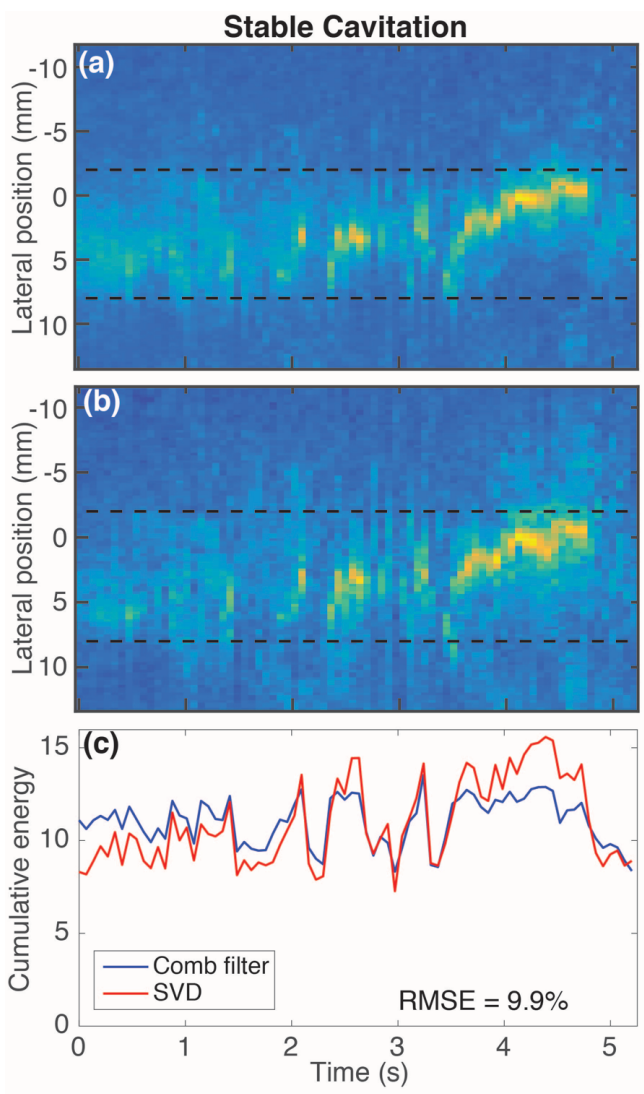

Figure 5. Spatio-temporal maps of cavitation energy (normalized to peak value) determined from measurements acquired in a tissue-mimicking gel phantom [1]. Stable cavitation energy using (a) the combfilter approach and (b) SVD-based processing. Inertial cavitation energy using (d) the comb-filter approach and (e) SVD-based processing. Spatial sum of harmonic energy in region bound between dashed lines for stable and inertial cavitation are shown in (c) and (f), respectively. RMSE: root-mean-squared error calculated as a percentage of the maximum energy determined using the comb-filter approach.

component.

\section{Passive cavitation maps}

Spatio-temporal maps of cavitation-noise energy (normalized to peak $V_{\text {RMS }}$ ) from data obtained in tissue-mimicking phantom are shown in Fig. 5. For stable and inertial cavitation, results indicate that the SVDbased map is in good qualitative agreement with the comb-filter-based map in spatiotemporal profile as well as the relative magnitude. Spatio-temporal profiles of stable and inertial cavitation energy were reduced to energy-time profiles by summing the energy near the focal region of the HIFU
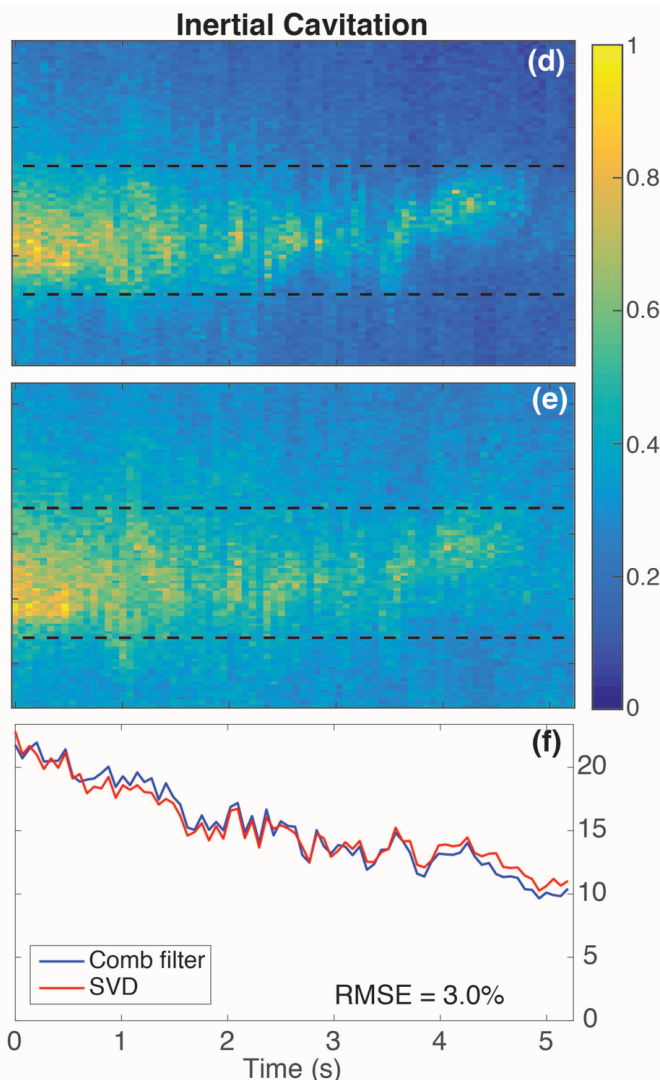

transducer (indicated by two horizontal black lines) and are shown in Figs. 5c and 5f. The root-mean-squared error (RMSE) between the energy-time profiles obtained using the two methods was $9.9 \%$ and $3 \%$ for stable and inertial cavitation, respectively. 
Maps of cavitation energy obtained using data from bovine liver sample degassed for 30 minutes are shown in Fig. 6. Stable and inertial cavitation maps obtained using the SVD-based filtering was in good maps obtained using the two filtering techniques on data from bovine liver sample degassed for 60 minutes (Fig. 7) also showed good agreement. The corresponding RMSE values for the energy-time curves for
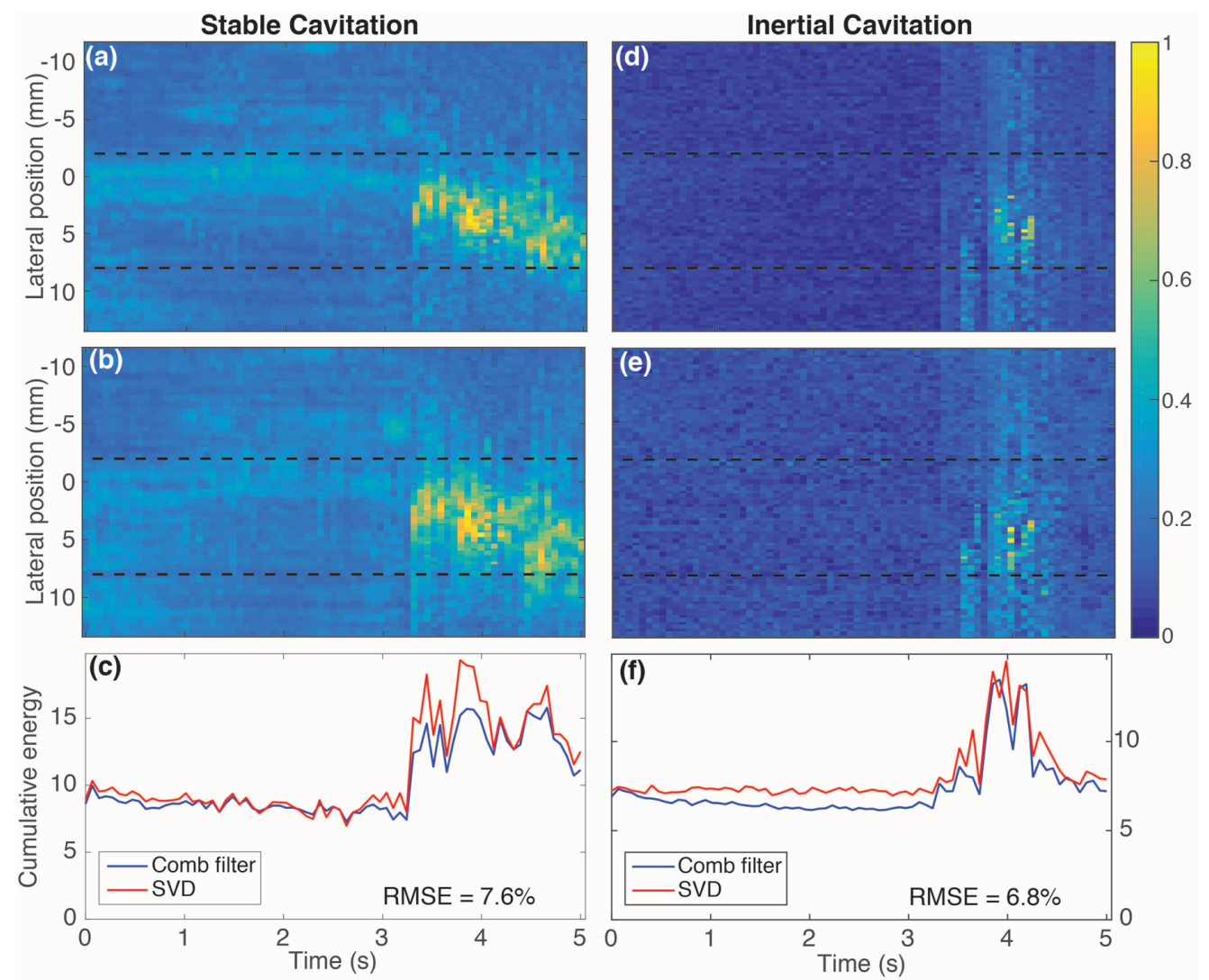

Figure 6. Spatio-temporal maps of cavitation energy (normalized to peak value) determined from measurements acquired in a bovine liver sample degassed for 30 minutes [1]. Stable cavitation energy using (a) the comb-filter approach and (b) SVD-based processing. Inertial cavitation energy using (d) the combfilter approach and (e) SVD-based processing. Spatial sum of harmonic energy in region bound between dashed lines for stable and inertial cavitation are shown in (c) and (f), respectively. RMSE: root-meansquared error calculated as a percentage of the maximum energy determined using the comb-filter approach.

qualitative and quantitative agreement with those obtained using the comb-filter approach. Spatio-temporal maps from both methods indicate that HIFU did not induce stable or inertial cavitation initially. The onset of cavitation occurred at $t>3 \mathrm{~s}$, and majority of the cavitation signal observed comprised of HIFU harmonics, indicating predominantly stable cavitation. The RMSE between the energy-time curves for stable and inertial cavitation obtained from this liver sample were $7.6 \%$ and $6.8 \%$, respectively. Similarly, cavitation-energy stable and inertial cavitation were $7.3 \%$ and $10 \%$, respectively.

\section{Discussion and Conclusion}

This study investigated the utility of SVD for analyzing and classifying cavitation activity detected by a linear ultrasound imaging array used as a passive cavitation detector. Specifically, the SVD-based approach was used to analyze previously acquired cavitation noise data [24] expressed as spatio-temporal maps of 
cavitation-noise energy associated with stable and inertial cavitation activity. SVDbased processing results were then compared directly against cavitation maps obtained previously using a comb-filter approach and reported in [24].
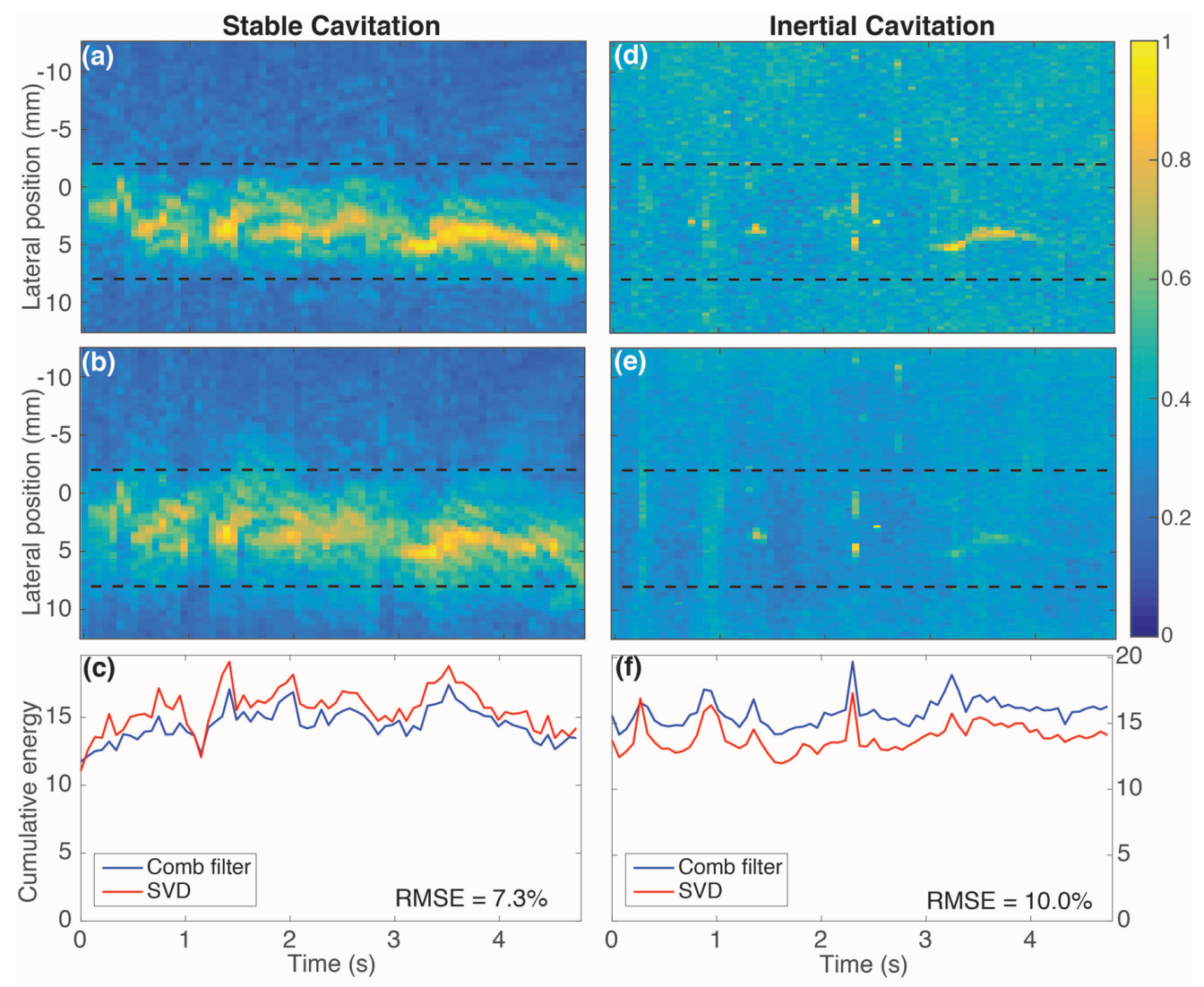

The SVD-based technique presented in this paper does not require a priori knowledge of the driving frequency of HIFU or its harmonics. Rather, the analysis of singular-vectors obtained from SVD of cavitation data was performed using a

Figure 7. Spatio-temporal maps of cavitation energy (normalized to peak value) determined from measurements acquired in a bovine liver sample degassed for 60 minutes [1]. Stable cavitation energy using (a) the comb-filter approach and (b) SVD-based processing. Inertial cavitation energy using (d) the combfilter approach and (e) SVD-based processing. Spatial sum of harmonic energy in region bound between dashed lines for stable and inertial cavitation are shown in (c) and (f), respectively. RMSE: root-meansquared error calculated as a percentage of the maximum energy determined using the comb-filter approach.

SVD-based analysis of cavitation was compared to the comb-filter approach using data from three experimental runs involving: 1) a tissue-mimicking gel phantom, 2) an excised bovine liver sample that was degassed for $30 \mathrm{~min}$, and 3) an excised bovine liver sample that was degassed for 60 min. The spatio-temporal maps of stable and inertial cavitation noise energy obtained using the two methods were in good agreement with each other for all three datasets (RMSE ranged from 3\% to $10 \%$ ). simple quantitative parameter, peak ratio, that effectively differentiated between singular-vectors that represented the harmonic components of the cavitation data from those that represented the broadband component of the cavitation data. Timedomain data of the desired cavitation type was separately obtained by simply projecting the unfiltered cavitation data on the appropriate singular-base. In this manner, the feasibility of SVD-based filtering described in this paper to achieve 
objective separation of stable cavitation from inertial cavitation was demonstrated. Therefore, SVD techniques could be extended to applications that employ multiple ultrasound transducers for simultaneously sonicating at different frequencies in order to improve control achieved over cavitation dynamics and improving therapeutic efficacy [25, 45-48]. In this regard, our approach overcomes a minor drawback associated with the combfilter approach, which is that it requires manual selection of HIFU fundamental and harmonic frequencies while designing the comb filter.

An SVD-based method is particularly robust in analyzing large data sets generated from similar experimental conditions. Decomposition of cavitation data onto a SVD-derived base of singular-vectors is statistically optimal in the sense that the greatest variance by any projection of the data is on the first singular-vector, the second greatest variance on the second singular-vector, etc. [38]. This implies that the singular-vectors that predominantly represent system noise will be ranked considerably lower than those representing cavitation events. Therefore, the proposed SVD-based analysis potentially is robust enough to process and filter cavitation data with relatively low signal-to-noise ratio because the analysis can be confined to relevant subset of singular-vectors (those that describe majority of the variance in the recorded cavitation signals).

However, an inherent weakness of the SVD approach is that it requires relatively large data sets for SVD-based analysis to be robust. Therefore, real-time implementation likely will require the use of ultra-fast ultrasound systems that operate a linear array (PCD) at frame rates exceeding 1000 frames/s. In this manner, a portion of the cavitation data can be sampled and processed while continuously acquiring additional data during sonication. Such an implementation of the SVD-based method can potentially enable ultra-high frame rate cavitation mapping, which could be particularly advantageous for monitoring and controlling a variety of therapeutic ultrasound applications that involve rapidly evolving cavitation fields, such as histotripsy $[11,14]$ and boiling histotripsy [49].

The present work was limited to three simplistic experimental scenarios. The SVDbased algorithm was "trained" using the gelphantom data, and then tested on cavitation data acquired using bovine-liver samples. Additional experimental studies, particularly where the system characteristics are learned using phantoms that better mimic acoustic and cavitation properties of tissue, are necessary to translate this methodology to practical applications. 


\section{Bibliography}

1. C.H. Farny, et al., The Correlation Between Bubble-Enhanced HIFU Heating and Cavitation Power. IEEE Transactions on Biomedical Engineering, 2010. 57: p. 175-184.

2. C.R. Jensen, et al., Spatiotemporal Monitoring of High-Intensity Focused Ultrasound Therapy with Passive Acoustic Mapping. Radiology, 2012. 262: p. 252-261.

3. J.A. Kopechek, et al., Cavitationenhanced MR-guided focused ultrasound ablation of rabbit tumors in vivo using phase shift nanoemulsions. Physics in Medicine and Biology, 2014. 59: p. 3465-3481.

4. D. Cranston, A review of high intensity focused ultrasound in relation to the treatment of renal tumours and other malignancies.

Ultrasonics Sonochemistry, 2015. 27: p. 654-658.

5. M. Dyson, Non-thermal cellular effects of ultrasound. The British journal of cancer. Supplement, 1982. 5: p. 165.

6. E. Neppiras, Acoustic cavitation series: part one: Acoustic cavitation: an introduction. Ultrasonics, 1984. 22(1): $\mathrm{p}$. 25-28.

7. N. McDannold, et al., Targeted disruption of the blood-brain barrier with focused ultrasound: association with cavitation activity. Physics in Medicine and Biology, 2006. 51: p. 793807.

8. C.D. Arvanitis, et al., Cavitationenhanced nonthermal ablation in deep brain targets: feasibility in a large animal model. Journal of Neurosurgery, 2015: p. 1-10.

9. L. Deng, et al., A multi-frequency sparse hemispherical ultrasound phased array for microbubble-mediated transcranial therapy and simultaneous cavitation mapping. Physics in Medicine and Biology, 2016. 61: p. 8476-8501.

10. S. Datta, et al., Correlation of cavitation with ultrasound enhancement of thrombolysis. Ultrasound in Medicine \& Biology, 2006. 32: p. 1257-1267.

11. A.D. Maxwell, et al., Noninvasive Thrombolysis Using Pulsed Ultrasound Cavitation Therapy - Histotripsy. Ultrasound in Medicine \& Biology, 2009. 35: p. 1982-1994.

12. F. Vignon, et al., Microbubble cavitation imaging. IEEE Transactions on Ultrasonics, Ferroelectrics and Frequency Control, 2013. 60: p. 661670.

13. K.B. Bader, et al., Shaken and Stirred: Mechanisms of Ultrasound-Enhanced Thrombolysis. Ultrasound in Medicine \& Biology, 2015. 41: p. 187-196.

14. E. Vlaisavljevich, et al., Histotripsyinduced cavitation cloud initiation thresholds in tissues of different mechanical properties. IEEE Transactions on Ultrasonics, Ferroelectrics, and Frequency Control, 2014. 61: p. 341-352.

15. V.A. Khokhlova, et al., Histotripsy methods in mechanical disintegration of tissue: Towards clinical applications. International Journal of Hyperthermia, 2015. 31: p. 145-162.

16. W. Yang and Y. Zhou, Effect of pulse repetition frequency of high-intensity focused ultrasound on in vitro thrombolysis. Ultrasonics Sonochemistry, 2017. 35: p. 152-160.

17. T. Li, et al., Pulsed High-Intensity Focused Ultrasound Enhances Delivery of Doxorubicin in a Preclinical Model of Pancreatic Cancer. Cancer Research, 2015. 75: p. 3738-3746.

18. F.E. Shamout, et al., Enhancement of Non-Invasive Trans-Membrane Drug Delivery Using Ultrasound and Microbubbles During Physiologically 
Relevant Flow. Ultrasound in Medicine \& Biology, 2015. 41: p. 2435-2448.

19. C. Crake, et al., Enhancement and Passive Acoustic Mapping of Cavitation from Fluorescently Tagged Magnetic Resonance-Visible

Magnetic Microbubbles In Vivo. Ultrasound in Medicine \& Biology, 2016. 42: p. 30223036.

20. C.D. Arvanitis, et al., Cavitationenhanced nonthermal ablation in deep brain targets: feasibility in a large animal model. Journal of Neurosurgery, 2016. 124: p. 1450-1459.

21. S.I. Madanshetty, et al., Acoustic microcavitation: Its active and passive acoustic detection. The Journal of the Acoustical Society of America, 1991. 90(3): p. 1515-1526.

22. R.A. Roy, et al., An acoustic backscattering technique for the detection of transient cavitation produced by microsecond pulses of ultrasound. The Journal of the Acoustical Society of America, 1990. 87(6): p. 2451-2458.

23. C.C. Coussios, et al., Role of acoustic cavitation in the delivery and monitoring of cancer treatment by high-intensity focused ultrasound (HIFU). International Journal of Hyperthermia: The Official Journal of European Society for Hyperthermic Oncology, North American Hyperthermia Group, 2007. 23: p. 105-120.

24. C.H. Farny, et al., Temporal and Spatial Detection of HIFU-Induced Inertial and Hot-Vapor Cavitation with a Diagnostic Ultrasound System. Ultrasound in Medicine \& Biology, 2009. 35: p. 603615.

25. H. Hasanzadeh, et al., Enhancement and control of acoustic cavitation yield by low-level dual frequency sonication: a subharmonic analysis. Ultrasonics sonochemistry, 2011. 18(1): p. 394-400.
26. J. Mamou and J.A. Ketterling, Subharmonic analysis using singularvalue decomposition of ultrasound contrast agents. The Journal of the Acoustical Society of America, 2009. 125: p. 4078.

27. M. Gyongy and C.-C. Coussios, Passive Spatial Mapping of Inertial Cavitation During HIFU Exposure. IEEE Transactions on Biomedical Engineering, 2010. 57: p. 48-56.

28. C.D. Arvanitis and N. McDannold, Integrated ultrasound and magnetic resonance imaging for simultaneous temperature and cavitation monitoring during focused ultrasound therapies. Medical Physics, 2013. 40: p. 112901.

29. V. Bull, et al., A Comparison of Acoustic Cavitation Detection Thresholds Measured with Piezo-electric and Fiberoptic Hydrophone Sensors. Ultrasound in Medicine \& Biology, 2013. 39: p. 2406-2421.

30. T. Li, et al., Passive Cavitation Detection during Pulsed HIFU Exposures of Ex Vivo Tissues and In Vivo Mouse Pancreatic Tumors. Ultrasound in Medicine \& Biology, 2014. 40: p. 1523-1534.

31. F. Prieur, et al., Observation of a cavitation cloud in tissue using correlation between ultrafast ultrasound images. IEEE Transactions on Ultrasonics, Ferroelectrics, and Frequency Control, 2015. 62: p. 12561264.

32. R.M. Jones, et al., Transcranial passive acoustic mapping with hemispherical sparse arrays using CT-based skullspecific aberration corrections: a simulation study. Physics in Medicine and Biology, 2013. 58: p. 4981-5005.

33. V.A. Salgaonkar, et al., Passive cavitation imaging with ultrasound arrays. The Journal of the Acoustical 
Society of America, 2009. 126(6): p. 3071-3083.

34. J. Gateau, et al., Combined passive detection and ultrafast active imaging of cavitation events induced by short pulses of high-intensity ultrasound. IEEE Transactions on Ultrasonics, Ferroelectrics and Frequency Control, 2011. 58: p. 517-532.

35. C.-y. Hsieh, et al., An Adaptive Spectral Estimation Technique to Detect Cavitation in HIFU With High Spatial Resolution. Ultrasound in Medicine \& Biology, 2011. 37: p. 1134-1150.

36. E. Lyka, et al., Sum-of-harmonics method for improved narrowband and broadband signal quantification during passive monitoring of ultrasound therapies. The Journal of the Acoustical Society of America, 2016. 140(1): p. 741-754.

37. B. Arnal, et al., In vivo real-time cavitation imaging in moving organs. Physics in Medicine and Biology, 2017. 62: p. 843-857.

38. J.E. Gentle, Numerical linear algebra for applications in statistics. 2012: Springer Science \& Business Media.

39. P.V. Chitnis, et al., Influence of shell properties on high-frequency ultrasound imaging and drug delivery using polymer-shelled microbubbles. IEEE Transactions on Ultrasonics, Ferroelectrics and Frequency Control, 2013. 60(1): p. 53-64.

40. C.H. Farny, Identifying and monitoring the roles of cavitation in heating from high-intensity focused ultrasound. 2007, Ph.D. thesis, Boston University, Boston, MA.

41. T. Leighton, The acoustic bubble. 2012: Academic press.

42. Y. Hao and A. Prosperetti, The dynamics of vapor bubbles in acoustic pressure fields. Physics of Fluids, 1999. 11(8): p. 2008-2019.
43. C.R. Thomas, et al., Dynamics and control of cavitation during highintensity focused ultrasound application. Acoustics Research Letters Online, 2005. 6(3): p. 182-187.

44. V.A. Khokhlova, et al., Effects of nonlinear propagation, cavitation, and boiling in lesion formation by high intensity focused ultrasound in a gel phantom. The Journal of the Acoustical Society of America, 2006. 119(3): p. 1834-1848.

45. R. Feng, et al., Enhancement of ultrasonic cavitation yield by multifrequency sonication. Ultrasonics sonochemistry, 2002. 9(5): p. 231-236.

46. S. Sokka, et al., Theoretical and experimental validation of a dualfrequency excitation method for spatial control of cavitation. Physics in medicine and biology, 2005. 50(9): p. 2167.

47. T. Ikeda, et al., Cloud cavitation control for lithotripsy using high intensity focused ultrasound. Ultrasound in medicine \& biology, 2006. 32(9): p. 1383-1397.

48. H.-L. Liu and C.-M. Hsieh, Singletransducer dual-frequency ultrasound generation to enhance acoustic cavitation. Ultrasonics sonochemistry, 2009. 16(3): p. 431-438.

49. Y.-N. Wang, et al., A multimodal evaluation of boiling histotripsy lesion properties in ex vivo bovine liver. 2016. 140(4): p. 3082-3082. 